\title{
CARTOGRAFÍA DE MODELOS DE COMBUSTIBLE CON TELEDETECCIÓN: APORTACIONES A UN DESARROLLO AMBIENTAL SOSTENIBLE
}

\author{
POR \\ DAVID RIAÑO, JAVIER SALAS Y EMILIO CHUVIECO
}

Los incendios forestales en el entorno mediterráneo:

el papel de la cartografía de los modelos de combustible

El bosque tiene gran variedad de funciones, como las de sumidero de $\mathrm{CO}_{2}$ y productor de $\mathrm{O}_{2}$, conservador del suelo (evita la erosión), nicho ecológico, fuente de recursos (leña, corcho, resina, frutos), uso recreativo, etc. Un desarrollo sostenible conlleva una utilización del bosque sin que pierda sus funcionalidades, para satisfacer la necesidades de la generación presente sin comprometer la capacidad de las generaciones futuras de satisfacer sus necesidades.

Los incendios forestales son procesos naturales muy ligados a los ecosistemas Mediterráneos, beneficiosos para su sostenibilidad, con el mantenimiento de la estructura de la vegetación y del paisaje. El principal factor que explica la incidencia de incendios en el clima Mediterráneo es el estrés hídrico de la vegetación en el verano, donde las altas temperaturas, unidas a las bajas precipitaciones, provocan la desecación de las especies vegetales (Salas, 1994). Las especies mediterráneas reducen el ritmo de evapotranspiración durante la estación seca. El bajo contenido de humedad, junto con estas esencias volátiles y combusticalá.

David Riaño, Javier Salas y Emilio Chuvieco. Dpto. de Geografía. Universidad de Al- 
bles, hacen que se pueda propagar el fuego con mayor facilidad. Condiciones climatológicas particulares pueden agravar esta situación, como son los fuertes vientos desecantes de componente continental, muy habituales en nuestro país (Pardos Carrión y González Alonso, 1991).

A estas peculiares condiciones naturales hay que añadir el papel fundamental que juega el fuego en nuestro entorno desde tiempos ancestrales. En el Neolítico ya se empleaba el fuego para favorecer la producción de alimentos y el pastoreo. La utilización histórica del fuego como herramienta agrícola, para la ganadería y silvicultura, implica una gran variedad paisajística y la presencia de comunidades vegetales altamente diversificadas. Esta situación no es equiparable a la de otras zonas con similar clima Mediterráneo. Por ejemplo, en California la vegetación presenta una gran continuidad, originándose incendios que abarcan grandes extensiones del territorio (Prieto, 1995).

La convivencia armónica entre el desarrollo y los incendios se ve truncada con los cambios que se producen en el siglo xx: incremento de la agricultura mecanizada, paso de la ganadería extensiva a ganadería estabulada, repoblación de grandes extensiones con especies pirófitas, disminución de la población rural, abandono de tierras, cese de actividades tradicionales (pastoreo, recogida de leña, corcho, resina, frutos, etc.), construcción de grandes vías de comunicación, y aumento de la presión turística y urbanística sobre el suelo forestal, principalmente (Pausas y Vallejo, 1999). Estos cambios nos hacen pensar que el desarrollo de este último siglo cuestiona la sostenibilidad en lo que se refiere a la gestión forestal. Se ha producido una homogeneización del paisaje y el aumento del combustible disponible, desembocando en un aumento exponencial del número de incendios en los últimos 40 años. Los incendios pasan a ser de pocos y pequeños a numerosos y, en ocasiones, de grandes dimensiones, con años especialmente críticos relacionados con veranos en los que la sequía fue más acuciante.

Las causas directas que provocan los incendios forestales son fundamentalmente antrópicas (un $95 \%$ de los casos conocidos), bien sea por negligencia, bien de manera intencionada. Los motivos que marcan estas actuaciones, que trastocan el aprovechamiento sostenible del bosque, obedecen a motivos muy diversos como serían la animadversión de ganaderos y pastores hacia las repoblaciones, rencillas personales, interés en atraer inversiones públicas relacionadas con la repoblación de

Estudios Geográficos, LXII, 243, 2001

$$
-310-
$$


la zona quemada, disconformidad de vecinos por intervenciones de la Administración Central, Regional o Local (Martín et al., 1998).

De esta forma, se reduce el periodo de recurrencia de los incendios, que idealmente se sitúa entre 50-100 años, llegando incluso a ciclos de 6-10 años, con lo que se ejerce una presión crítica sobre la vegetación. Se produce un ciclo de realimentación negativa en el que se priman los estadios más pioneros y la adaptación de las especies pirófitas (Prieto, 1995) que a su vez conllevan una mayor probabilidad de incendio. $\mathrm{Al}$ disminuir la cobertura vegetal, hace que se disminuya la capacidad de retención del agua en el suelo, lo que implica una menor infiltración y consecuentemente una mayor escorrentía superficial, lo que aumenta el riesgo de erosión y el avance de la desertificación.

En la propagación del incendio forestal influyen las características de la vegetación, el viento reinante y las condiciones topográficas. En lo que se refiere a la vegetación, resulta clave considerar su estado hídrico y estructura (ICONA, 1990). El primer factor condiciona la facilidad de ignición, y el segundo la transmisión del calor y la cantidad de combustible disponible. La combustibilidad de una formación vegetal depende de un elevado número de factores: cantidad, particulación, compacidad, continuidad, densidad, etc. Medir todas estas propiedades es un proceso demasiado lento para hacer predicciones de incendios; por ello, se ha optado por utilizar modelos de combustible que clasifican las formaciones vegetales de acuerdo a esas características. De esta manera se definen como una representación matemática del estrato combustible, con la inclusión de todos los parámetros necesarios para calcular ciertas variables relacionadas con el comportamiento del fuego, que normalmente son la velocidad de propagación y la intensidad del fuego (Deeming, 1975; Rothermel, 1972). Las características a tener en cuenta a la hora de definir un modelo están relacionadas con la altura y densidad de la vegetación.

A inicios de los 80, el Servicio Forestal de los Estados Unidos generó una tipología de modelos de combustible dentro del programa de modelización del fuego BEHAVE. Esta clave ha sido ampliamente utilizada y ha servido como base a la creación de otras nuevas (Anderson, 1982; Burgan y Rothermel, 1984). Consta de 13 modelos, que se agrupan en cuatro categorías en función de cuál es el elemento propagador del fuego (considerando incendios de superficie): pastizal, matorral, hojarasca y restos de corta. En el caso europeo, y más concretamente en la 
Europa Mediterránea, se ha diseñado una tipología, que basándose en principios similares ha obtenido una caracterización más acorde con nuestra vegetación; se trata del proyecto Prometheus (Prometheus, 1999), que describe siete modelos de combustible, considerando tres elementos de propagación del fuego: pastizal, matorral y hojarasca.

La cartografía de modelos de combustible aporta una información fundamental para los sistemas de prevención, predicción y modelado de incendios. Un buen conocimiento y una buena representación espacial de estos modelos resulta de máximo interés para llevar a cabo las siguientes actividades:

- Planificación del sistema de prevención de incendios: tratamiento de la vegetación (entresaca selectiva, quemas controladas).

- Planificación del sistema de detección de incendios: localización de las torretas de vigilancia, vuelos de vigilancia, etc.

- Planificación del sistema de extinción de incendios: localización de retenes de incendios, maquinaria para la extinción, etc.

- Estimación del peligro y riesgo de incendio.

- Simulación del comportamiento del fuego.

- Simulación de los efectos de los incendios.

- Simulación de la emisión y dispersión de gases provenientes de los incendios a la atmósfera.

Tradicionalmente, la mayor parte de los recursos económicos para combatir los incendios forestales se han consumido en extinción, para disminuir el daño cuando se inicia el fenómeno, mientras que la inversión en prevención ha recibido mucho menor interés. Las políticas actuales van un poco más encaminadas a la mejora de los sistemas de prevención, predicción y detección. En el Plan Nacional de Investigación Científica, Desarrollo e Innovación Tecnológica para el periodo 20002003 se establece como línea prioritaria para el año 2000 el desarrollo de nuevas técnicas de previsión y prevención de episodios de incendios forestales (http://www.seui.mec.es/). En cuanto a los nuevos sistemas de detección, es interesante mencionar la potencialidad de las cámaras de infrarrojos (IR), como sistema terrestre de detección precoz (Aranda et al., 1999). En lo que se refiere a los sistemas remotos de detección hay que destacar el proyecto FUEGO, englobado en el IV Programa Marco de la Unión Europea «Medio Ambiente y Clima», donde participa, entre otros, el Ministerio de Medio Ambiente. Este proyecto prevé lan-

Estudios Geográficos, LXII, 243, 2001

$$
-312-
$$


zar una constelación de minisatélites que permita detectar incendios, transcurridos menos de 15 minutos desde el origen del fuego (http://www.insa.es/fuego/default.htm). Además, se está invirtiendo dinero nacional y europeo en la mejora de los programas de simulación del comportamiento del fuego, que mejorarán también las tareas de combate y extinción.

El objetivo de este trabajo es la elaboración de una metodología útil para la cartografía de modelos de combustible que pueda ser extrapolable a otras áreas de estudio, con las ventajas que supone el empleo de la teledetección: bajo coste y cobertura global. El mapa de modelos de combustible generado deberá poder ser integrado en los sistemas de prevención, predicción y modelado de incendios. Los objetivos específicos de nuestro trabajo se concretaron en:

- Identificación de las bandas Landsat-TM con mayor capacidad para discriminar modelos, incluyendo tanto las originales, como las derivadas (texturales), y de la información auxiliar generada a partir del modelo digital del terreno (altitud, pendiente e iluminación).

- Evaluación de la importancia de las correcciones radiométrica y topográfica en los resultados de la clasificación digital.

Cartografía de modelos de combustible con teledetección: revisión bibliográfica

La teledetección espacial puede servir como tecnología geográfica para la cartografía de modelos de combustibles, presentando ciertas ventajas sobre otro tipo de métodos (Salas y Chuvieco, 1995):

- Resulta fácilmente actualizable, gracias a la repetitividad de la observación espacial.

- Es más económica que la fotografía aérea, cuando estamos trabajando sobre grandes espacios.

- Permite ofrecer una cobertura global.

- Dispone de información en regiones no visibles del espectro (infrarrojo cercano, infrarrojo medio, infrarrojo térmico, microondas).

- Los datos se pueden gestionar e interpretar digitalmente, lo que permite realizar operaciones complejas, costosas o inaccesibles al análisis visual (Chuvieco, 1996) y una buena conexión con otras variables espaciales.

Estudios Geográficos, LXII, 243, 2001

$$
-313-
$$


El principal problema, inherente también a la foto aérea, es la dificultad para identificar el substrato inferior, situado bajo los árboles, de importancia vital para determinar el comportamiento del fuego en incendios de superficie (Salas y Chuvieco, 1995; Salazar, 1982). Otros problemas de la teledetección espacial, comparativamente menos relevantes, son la estimación de las diferentes alturas de la vegetación y las correcciones radiométricas del efecto topográfico y atmosférico.

El trabajo pionero de Kourtz (1977) introdujo las principales técnicas de clasificación digital que se utilizarían en los años siguientes: clasificación supervisada (algoritmo de máxima probabilidad), no supervisada y componentes principales. Además, empleó imágenes de distintas fechas para retener la variabilidad temporal de los modelos de combustible. En la misma línea, Rabii (1979) obtuvo una clasificación no supervisada de trece modelos de combustible en la que destacó la capacidad de las bandas Landsat MSS-5 y MSS-7 (rojo e infrarrojo cercano, respectivamente) para discriminar la vegetación. En este trabajo se exponía la necesidad de que las diferencias de iluminación fueran normalizadas, de cara a obtener una clasificación más precisa. El interés de abordar esta corrección topográfica de las imágenes se ha resaltado también por muchos otros autores (Cosentino et al., 1981; Salas y Chuvieco, 1995; Salazar, 1982; Stow, 1993; Tymstra y Ellehoj, 1994; Yool et al., 1984) en el ámbito de la cartografía de combustibles forestales.

En el marco del proyecto FIRESCOPE, que surgió a consecuencia de una campaña especialmente catastrófica de incendios forestales en California, se realizaron importantes avances en la cartografía de modelos combustibles (Cosentino y Estes, 1981; Cosentino et al., 1981; Yool et al., 1984). Se comenzó a emplear la textura como variable auxiliar sobre la banda roja del Landsat MSS. En ese caso, la textura se definió calculando la desviación típica local, en una ventana de $3 \times 3$ píxeles que se desplaza sucesivamente sobre la imagen. Los métodos de clasificación empleados son muy variados, desde clasificación supervisada a no supervisada, así como las variables de partida, que junto a las propias bandas del sensor incluyen la ocurrencia histórica del fuego, la precipitación y la topografía. Estos dos últimos parámetros se incorporaron en un intento de modelizar la disponibilidad de agua e indirectamente la biomasa.

Shasby et al. (1981) propusieron una clasificación mixta similar a la del proyecto FIRESCOPE con una imagen Landsat MSS en la que se introducía la altitud, pendiente y orientación como variables auxiliares. Se 
basó en la idea de que las plantas se distribuyen en función de posiciones topográficas. A partir de este trabajo, resulta frecuente introducir la altitud en la discriminación de modelos (Burgan y Shasby, 1984; Miller et al., 1986; Miller y Johnston, 1985; Root et al., 1986; Salas y Chuvieco, 1995; Van Wangtendonk, 1997; Vasconcelos et al., 1998; Werth et al., 1985).

En el trabajo de Salazar (1982) se remarcó el interés de utilizar los índices de vegetación, principalmente el verde/rojo y el infrarrojo/rojo. Esta autora identifica tres factores que afectan a la reflectividad de los modelos de combustible: el dosel vegetal, el suelo y la topografía. Dichos factores guardan relación con los componentes puros de vigor vegetal, suelo y sombra que utilizan posteriormente Roberts et al. (1997), junto con el mapa de vegetación obtenido a partir del análisis de mezclas espectrales, para obtener el mapa de combustibles. Esta idea de generar primero un mapa de vegetación y, posteriormente, la cartografía de los modelos de combustible se emplea también por muchos otros autores (Agee y Pickford, 1985; Benson et al., 1982; Burgan et al., 1998; Roberts et al., 1997; Root et al., 1986). El mapa de vegetación se complementa con datos auxiliares (ocurrencia histórica del fuego, mapa de suelos, altitudes, trabajo de campo, etc.) para obtener el mapa de modelos de combustible.

El análisis multitemporal puede servir como herramienta para solucionar problemas de confusión entre combustibles (Dixon et al., 1984), no ya sólo analizando el estado de la vegetación en distintas estaciones del año, sino también recogiendo la variabilidad interanual. Esta técnica es de interés para la cartografía de modelos de combustible tanto con sensores de baja resolución espacial como el NOAA-AVHRR, como con imágenes de mayor resolución. Las imágenes AVHRR, aunque no proporcionan una descripción muy detallada del territorio, sí son eficaces para cubrir grandes espacios, gracias a su elevada frecuencia temporal. Miller y Johnston (1985) y Miller et al. (1986) utilizaron el índice de vegetación normalizado (NDVI) de imágenes de varias fechas para diferenciar once modelos mediante una clasificación no supervisada. McKinley et al. (1985), usando una técnica similar, realizaron una clasificación de los modelos de combustible para once estados de USA. Este trabajo lo ha continuado más recientemente Burgan et al. (1998) utilizando, además de la serie temporal del NDVI, información auxiliar y extensivo trabajo de campo.

Estudios Geográficos, LXII, 243, 2001 
En cuanto a las imágenes de alta resolución espacial, el análisis temporal se ha empleado por varios autores (Stow, 1993;Van Wangtendonk, 1997; Van Wangtendonk y Root, 1999). Además del análisis temporal, las líneas de trabajo se han orientado hacia el empleo de nuevas técnicas de clasificación, como las redes neuronales (Vasconcelos et al., 1998).

La mayoría de los estudios consultados sólo utilizan las bandas de la región visible y los infrarrojos cercano y medio, si bien, puede resultar interesante el uso de otras regiones del espectro. En general, los autores no consideran al gradiente de temperatura como una capa de interés temático para discriminar combustibles (Salas y Chuvieco, 1995) y por lo tanto el canal térmico se ve excluido a la hora de realizar la clasificación. Sin embargo, Agee y Pickford (1985) resaltan la importancia de este factor a la hora de diferenciar los principales tipos de combustible. Por otro lado, existen varios trabajos que proponen el uso de las microondas. Kyun (1997) compagina el radar (ERS1-SAR) y los datos ópticos del TM, con la idea de introducir información sobre la estructura de la vegetación. Los resultados de su clasificación supervisada no mejoran sobre los que tienen en cuenta sólo la imagen TM, debido en principio a la dificultad de corregir el efecto topográfico en las imágenes radar.

Finalmente, conviene destacar el reciente empleo de los sensores hiperespectrales aerotransportados, como el AVIRIS, que abren nuevas posibilidades a la cartografía de modelos de combustibles (Roberts $e t$ al., 1997).

\section{Área de estudio}

Para mostrar la capacidad de la teledetección en la cartografía de modelos de combustible, presentaremos un ejemplo llevado a cabo en el Parque Nacional de Cabañeros.

Se encuentra al nordeste de la provincia de Ciudad Real y sudoeste de la de Toledo. Toda esta área geográfica, que forma parte de la comarca que se ha denominado históricamente como «Comarca de los Montes Norte», constituye un amplio agrosistema de características singulares, no sólo por sus componentes naturales, sino por las características de su explotación tradicional, la cual ha conformado el actual paisaje de la zona.

Estudios Geográficos, LXII, 243, 2001

$$
-316-
$$


Dentro del conjunto de los Montes Norte, la organización del relieve se basa en estructuras geológicas y materiales antiguos plegados, metamorfizados e intruidos por rocas graníticas como consecuencia de la orogenia hercínica, que tras la actuación de procesos de modelado posteriores han generado distintas formas estructurales que se repiten, dando lugar a tres grandes tipos de unidades morfológicas naturales: las sierras, las depresiones y las llanuras (Alonso et al., 1997).

El clima de Cabañeros es mediterráneo, templado y semiárido, con una amplitud térmica próxima a los $20^{\circ} \mathrm{C}$, característica propia de los climas continentalizados. La temperatura media está entre los $13^{\circ} \mathrm{C}$ y los $16^{\circ} \mathrm{C}$ (Alonso et al., 1997) de acuerdo con la variabilidad altitudinal.

El piso bioclimático predominante en Cabañeros es el mesomediterráneo, que debido a la diferencia de precipitaciones permite establecer la dominancia de ombroclimas seco, con presencia de encinares como vegetación potencial, y subhúmedo, con alcornocales y melojares. El piso supramediterráneo se alcanza sólo en altitudes superiores a los 1.100-1.200 m, donde aparecen melojares. Cabañeros pertenece a la provincia biogeográfica luso-extremadurense, estando presentes las siguientes series climatófilas de vegetación (Peinado y Martínez, 1985):

- Serie mesomediterránea silicícola de la encina.

- Serie mesomediterrána subhúmeda silicícola del alcornoque.

- Serie mesomediterránea húmeda silicícola del roble melojo.

- Serie supramediterránea silicícola del roble melojo.

A éstas hay que añadir la geoserie riparia de los suelos silíceos lusoextremadurenses, que no está caracterizada en función del clima sino por las condiciones de humedad y salinidad del suelo.

La vegetación climax, que da el nombre a la serie (encinar, alcornocal y melojar), es la etapa madura, no dándose ninguna tendencia a la sucesión natural. Las etapas de sustitución o subseriales responden a la dominancia del matorral, mientras que las comunidades permanentes están formadas por herbáceas. Desde el punto de vista de los modelos de combustible, no interesa tanto estudiar la vegetación en función de las series, sino en función de la etapa de maduración en la que se encuentra el ecosistema, es decir, formaciones arbóreas, arbustivas o pastizales.

Estas especies autóctonas han sido sustituidas por repoblaciones forestales, fundamentalmente de pino resinero (Pinus pinaster), en mu-

Estudios Geográficos, LXII, 243, 2001 
chas partes del Parque Nacional, que son administradas como explotaciones forestales. Esta especie es pirófita, degrada el suelo y no se regenera tras los incendios como las especies autóctonas presentes en la zona.

Cartografía de modelos de combustible

Metodología.-El proceso seguido para obtener la clasificación digital de los modelos de combustible fue el siguiente (figura 1):

- Selección de las imágenes de satélite para la clasificación.

- Generación del modelo digital de elevaciones (MDE) y sus derivados (pendiente e iluminación) para ser utilizados en el proceso de co-

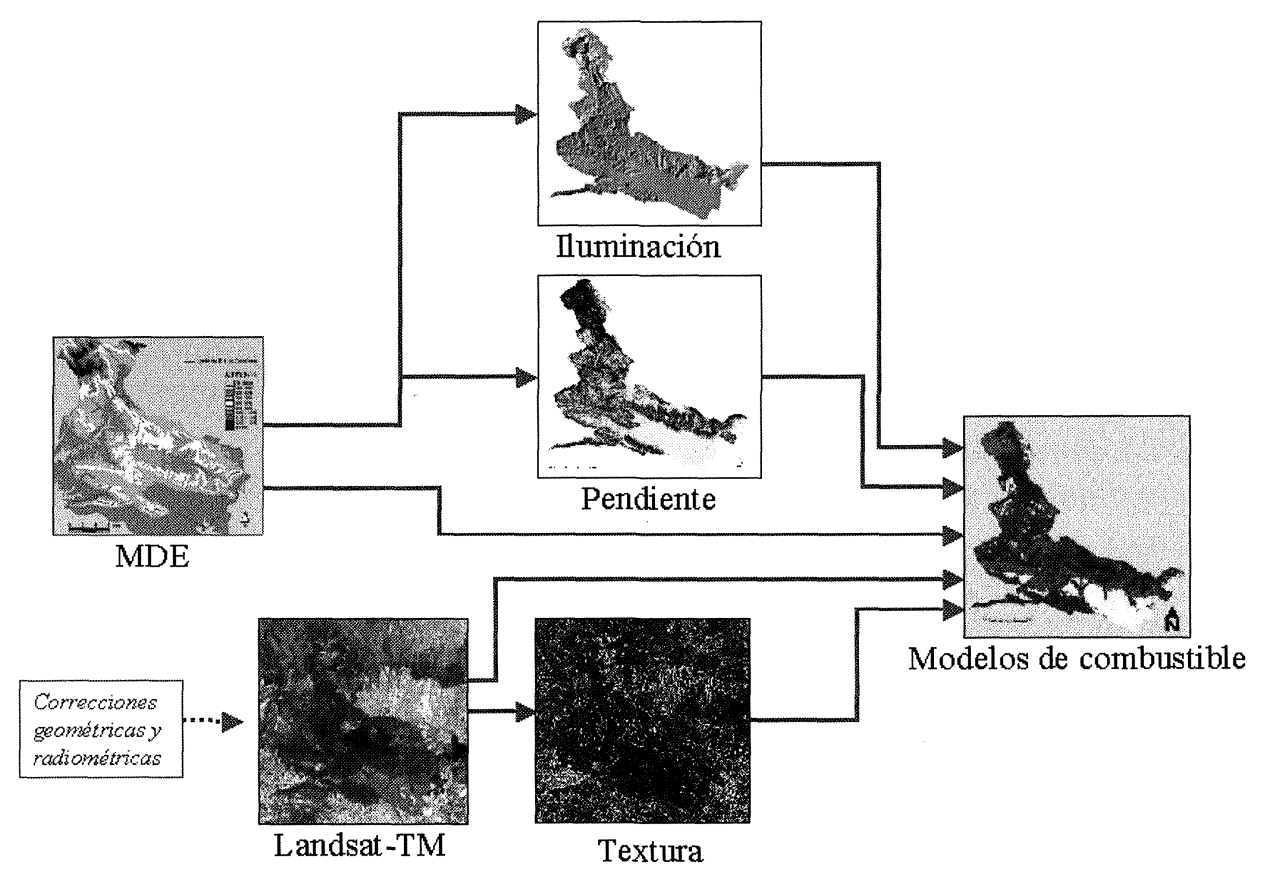

Fig. 1.-Esquema de la metodología desarrollada para la cartografía de modelos de combustible.

Estudios Geográficos, LXII, 243, 2001

$$
-318-
$$


rrección topográfica y como capas de información auxiliar para la clasificación.

- Procesado de las imágenes: corrección geométrica, del efecto atmosférico y del efecto topográfico.

- Realización de trabajo de campo para llevar a cabo la selección de campos de entrenamiento en la clasificación digital y para su posterior verificación.

- Proceso de clasificación digital mediante el algoritmo de máxima probabilidad.

Generación del MDE.-La generación del MDE se realizó partiendo de la digitalización manual de los mapas del Servicio Geográfico del Ejército a escala 1:50.000, con equidistancia de $20 \mathrm{~m}$ entre las curvas de nivel, para todo el Parque Nacional de Cabañeros y su perímetro de protección. La interpolación espacial para generar el MDE raster se llevó a cabo mediante el algoritmo de distancia transformada (Rosenfeld y Pfaltz, 1966). Dicho algoritmo se basa en el método de la interpolación lineal. Como paso previo se genera una curva de nivel intermedia entre cada dos curvas de nivel contiguas. Finalmente, se realiza la interpolación lineal empleando las curvas intermedias y las originales. Este algoritmo minimiza el problema de collados o puntos de inflexión. El resultado se verificó mediante un muestreo sistemático de toda la zona interpolada, obteniéndose un error promedio de $\pm 9 \mathrm{~m}$. Finalmente, el MDE se utilizó para generar los mapas de pendiente e iluminación.

Selección y corrección de las imágenes.-Para recoger la variabilidad fenológica de las especies, se eligieron dos imágenes Landsat-TM, una de primavera ( 5 de mayo de 1998) y otra de verano ( 21 de julio de 1997), libres de nubes y con unas buenas condiciones de iluminación (por la elevada altura solar), con el fin de reducir el efecto topográfico en la medida de lo posible.

La corrección geométrica se realizó utilizando como referencia los mapas del Servicio Geográfico del Ejército a escala 1:50.000. Se localizaron 40 puntos de control, distribuidos homogéneamente en zonas no sujetas a dinamismo temporal, ajustándose una función de transformación de $2^{\circ}$ grado entre la imagen sin corregir y el mapa de referencia. Finalmente, se transfirieron los valores digitales de la imagen a la nueva posición mediante una interpolación bilineal. Una vez que se tuvo corregida una imagen se utilizó ésta como referencia y se realizó la corrección de

Estudios Geográficos, LXII, 243, 2001

$$
-319-
$$


la otra fecha, lo que aseguró una buena superposición de ambas imágenes. El error, en ambos casos, se cifró en torno a 0,5 píxeles.

La corrección atmosférica sirvió para paliar el efecto de la dispersión de la radiación electromagnética originada por parte de los gases y partículas en suspensión de la atmósfera, para que las variaciones en la reflectividad observada de los modelos de combustible fueran independientes de las condiciones de adcquisición. El método de corrección aplicado fue el de la transmisividad de la atmósfera descendente por defecto (Chavez, 1996; Riaño, 2000).

Como resultado de la aplicación de la corrección atmosférica sobre las imágenes de satélite se verificó que la reflectividad de las dos imágenes era comparable entre sí al presentarse en ambos casos las siguientes características (PCI, 1997):

- La vegetación presenta un pico de reflectividad en el verde.

- La reflectividad del agua está entre 0-1\% en las bandas TM4, 5 y 7.

- La reflectividad del suelo desnudo y del cemento debe incrementarse con el aumento de la longitud de onda.

Para la corrección topográfica se eligió un método semiempírico conocido como corrección-c (Teillet et al., 1982; Riaño, 2000). Dicha corrección tiene en cuenta el comportamiento no lambertiano de la superficie terrestre, es decir que la luz solar no se refleja con la misma intensidad en todas las direcciones. La corrección aplicada permitió compensar las diferencias de iluminación solar debidas a la variación en la exposición del terreno. Las zonas de umbría presentaban menos reflectividad de lo que debieran, mientras que las zonas en solana presentaban una reflectividad más alta de lo esperado. Tras realizar la corrección topográfica se verificó que se habían eliminado ciertas sombras que aparecían en las imágenes por la baja iluminación (figura 2). De esta manera se consiguió homogeneizar la variabilidad espectral dentro de cada uno de los modelos de combustible.

Trabajo de campo.-El trabajo de campo consistió en seleccionar 102 parcelas homogéneas en cuanto al modelo de combustible se refiere, identificando dicho modelo de combustible en función de una clave fotográfica, cuyo esquema aparece en la figura 3. Pretendimos que las parcelas estuvieran bien distribuidas en la zona de estudio y representaran adecuadamente la variación espacial de cada uno de los modelos. La

Estudios Geográficos, LXII, 243, 2001

$$
-320-
$$




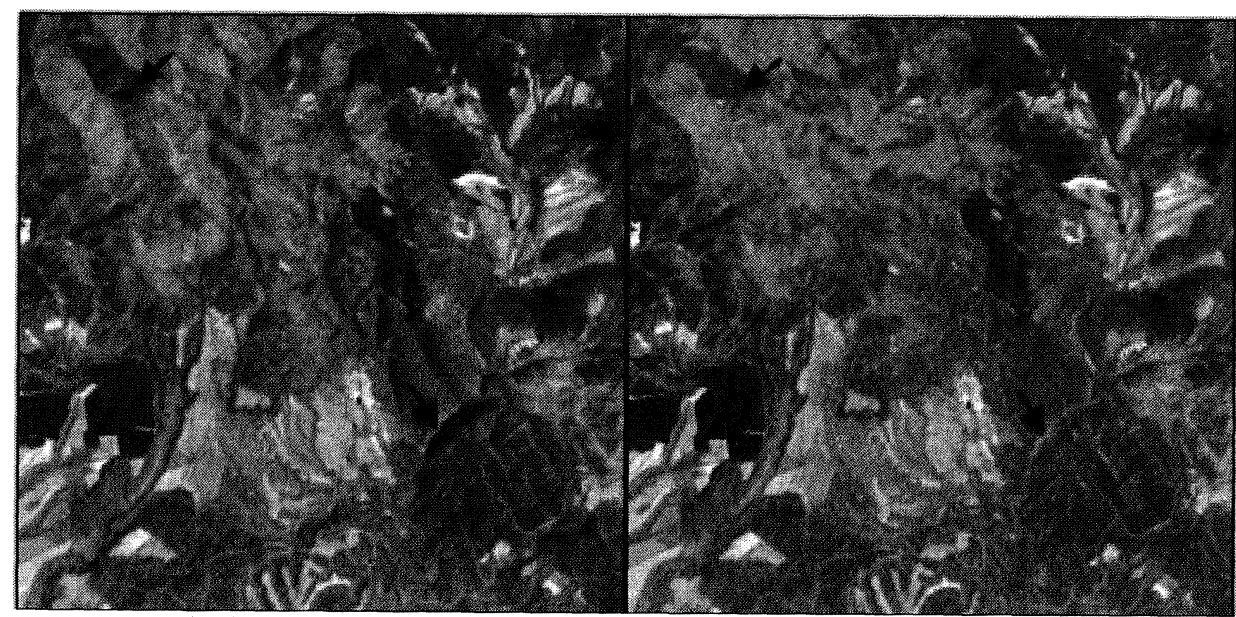

FIG. 2.-Detalle de la zona norte del Parque Nacional de Cabañeros antes (izquierda) y después de realizar la corrección topográfica (derecha). Las flechas señalan ejemplos de zonas donde la corrección topográfica ha sido eficiente.

Imagen del día 21-7-97.

identificación se realizó según la descripción de los siete modelos de combustible propuestos en el proyecto europeo Prometheus (Prometheus, 1999):

- Modelo 1: Pasto.

- Modelo 2: Matorral (0.30-0.60 m).

- Modelo 3: Matorral (0.60-2.00 m).

- Modelo 4: Matorral (2.00-4.00 m).

- Modelo 5: Bosque sin combustibles de superficie (sin arbustos ni herbáceas).

- Modelo 6: Bosque con combustibles de superficie. Existe diferencia de altura entre los arbustos y las copas de los árboles.

- Modelo 7: Bosque con combustibles de superficie. No existe diferencia de altura entre los arbustos y las copas de los árboles (continuidad vertical).

Cada parcela se localizó mediante un GPS tipo GARMIN, que ofrece una precisión de $\pm 100 \mathrm{~m}$. Se distinguieron varias comunidades vegetales dentro de cada modelo. Además, se localizaron parcelas con pedri- 


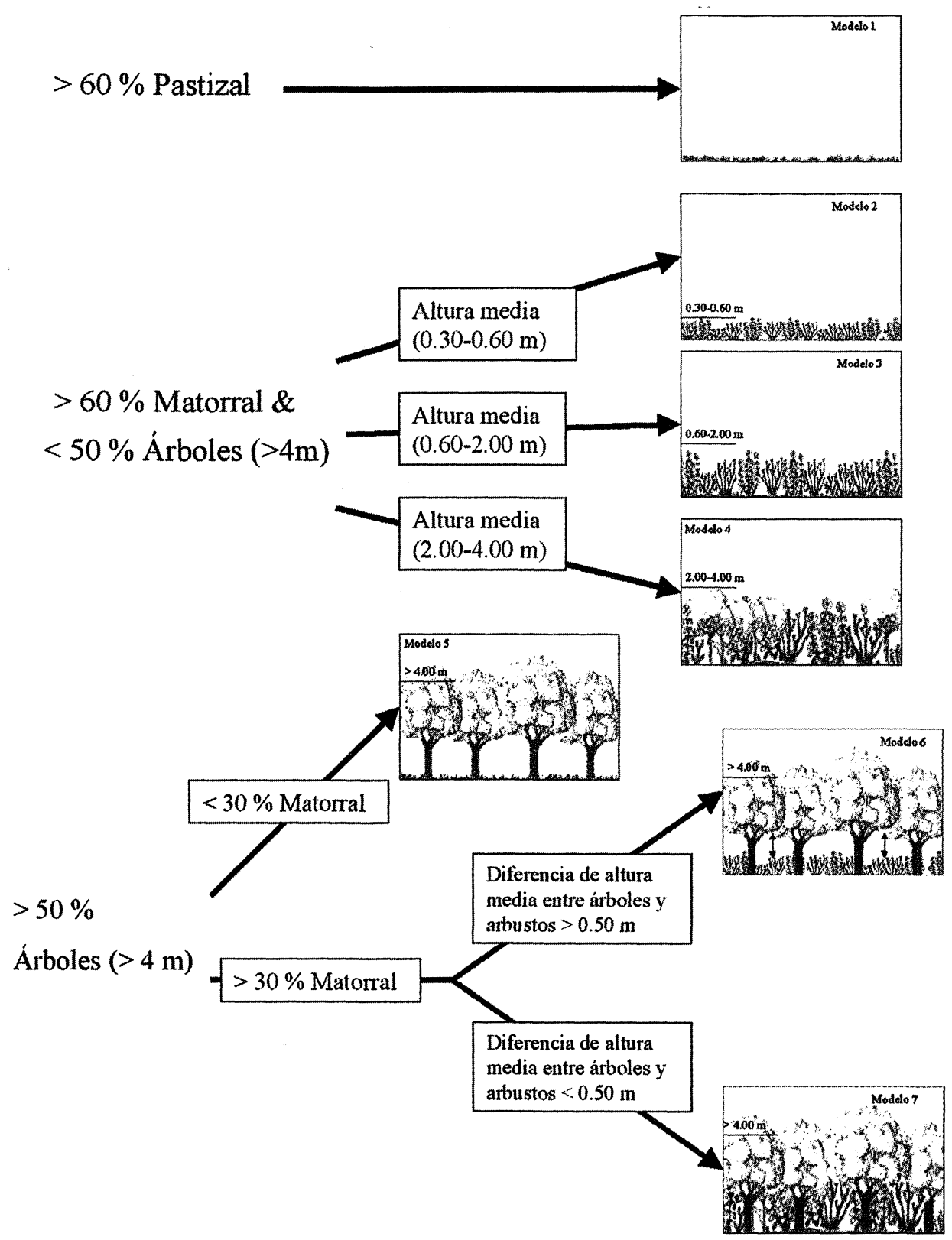

FIG. 3.-Esquema de modelos de combustible del proyecto Prometheus.

Estudios Geográficos, LXII, 243, 2001

$$
-322-
$$


zas y se identificó sobre las imágenes el agua y el suelo desnudo. Se anotó para cada parcela las principales especies, la densidad, la cobertura, la altura, la pendiente, el ángulo de incidencia solar, las coordenadas y la fecha de adquisición.

Clasificación supervisada de la imagen.-Se realizó una clasificación digital supervisada contando con datos espectrales y espaciales, provenientes de las imágenes de satélite, e información auxiliar, extraída del MDE.

En cuanto a los datos reflectivos se refiere, se excluyó la banda TM 7 de ambas imágenes ya que su información redundante hacía empeorar los resultados de la clasificación. La banda TM6 del infrarrojo térmico se transformó en valor de temperatura de superficie mediante la inversión de la ecuación de Planck (Wukelic et al., 1989).

La variabilidad espacial se evaluó mediante una banda de textura, que mide la homogeneidad en la banda TM3 en una ventana móvil de $3 \times 3$ píxeles, mediante el cálculo de la matriz de coincidencias (PCI, 1997). En esta matriz se miden las veces que un determinado valor digital coincide con otro. La textura será lisa cuando la frecuencia sea alta en valores digitales similares. Seleccionamos la banda TM3 para el cálculo de texturas, pues proporciona una mayor variabilidad espacial, siendo ampliamente empleada en la cartografía de modelos de combustible (Cosentino y Estes, 1981; Cosentino et al., 1981; Salas y Chuvieco, 1995; Yool et al., 1984).

Como información auxiliar se incluyó en el proceso de clasificación el MDE y las variables derivadas de éste, como son la pendiente y la iluminación.

A la hora de definir los campos de entrenamiento hay que intentar que cada uno de los modelos de combustible presente unas características espectrales homogéneas, de tal manera que no se confunda con el resto de modelos de combustible. Con este propósito se diferenciaron 19 categorías con ND homogéneos, en función fundamentalmente de los distintos tipos de vegetación. Las clases espectrales, tras la fase de asignación, se agruparon en los 7 modelos de combustible de la leyenda Prometheus que pretendíamos incluir en el mapa resultado.

La selección de campos de entrenamiento y la posterior verificación de la cartografía se apoyaron en el trabajo de campo realizado. Se consideró de máximo interés incluir en la clasificación todos los campos muestreados en terreno, ya que de este modo se recogía toda la varia-

Estudios Geográficos, LXII, 243, 2001

$$
-323-
$$


bilidad existente entre las clases y dentro de cada una de ellas (diferentes exposiciones, pendientes, densidades, etc.). Los alrededores de cada una de las parcelas de muestreo se utilizaron como campo de entrenamiento, mientras que la zona central (9 píxeles) de estas parcelas de muestreo se empleó en la verificación. Por motivos logísticos, se optó por esta solución intermedia entre utilizar los campos de entrenamiento o muestrear nuevas áreas test para la verificación de los resultados. De este modo, aunque los resultados de la verificación se verán algo afectados por el fenómeno de autocorrelación espacial, el sesgo será muy inferior al que se produciría en el caso de utilizar los campos de entrenamiento. Para ensayos posteriores están previstas nuevas salidas de campo que permitan un muestreo exclusivo para la fase de verificación.

El algoritmo empleado para la asignación fue el de máxima probabilidad, asignándose cada píxel a la categoría más probable, considerando como espacio de medida el formado por todas las bandas de entrada.

Resultados. - Los resultados de la clasificación con la imagen de verano, utilizando las reflectividades de las bandas TM 1-5, ofrecieron una fiabilidad global del $58 \%$. Al utilizar las imágenes de primavera y verano la precisión aumentó hasta el $67 \%$. Este resultado mejoró al 79 $\%$ cuando se introdujeron datos de altitud, pendiente e iluminación. Finalmente, al incluir la textura y las bandas térmicas (TM6) la precisión llegó a ser del $83 \%$ (figura 4).

El proceso de clasificación proporcionó la distinción de las 19 categorías espectrales que se agruparon en los 7 modelos finales. El mapa resultante se suavizó mediante un filtro modal, asignando a cada píxel el valor de la moda de una ventana móvil de $3 \times 3$ píxeles.

En la cartografía resultante de la clasificación se puede observar como la zona norte de Cabañeros está dominada por modelos de arbolado 5, 6 y 7 . El modelo 7 prevalece, sobre todo, en las zonas de densidad no muy alta de arbolado, donde puede llegar la luz para el crecimiento del matorral. El modelo 5 se corresponde normalmente con las zonas de repoblación o vegetación autóctona donde se han llevado a cabo trabajos forestales y pastoreo o donde el avanzado estado de la sucesión impide el paso de la luz necesaria para el desarrollo del sotobosque. El modelo 6 responde a zonas donde llega luz suficiente y se ha producido el abandono de la actividad forestal. El modelo 3 domina la mayor parte de

Estudios Geográficos, LXII, 243, 2001 


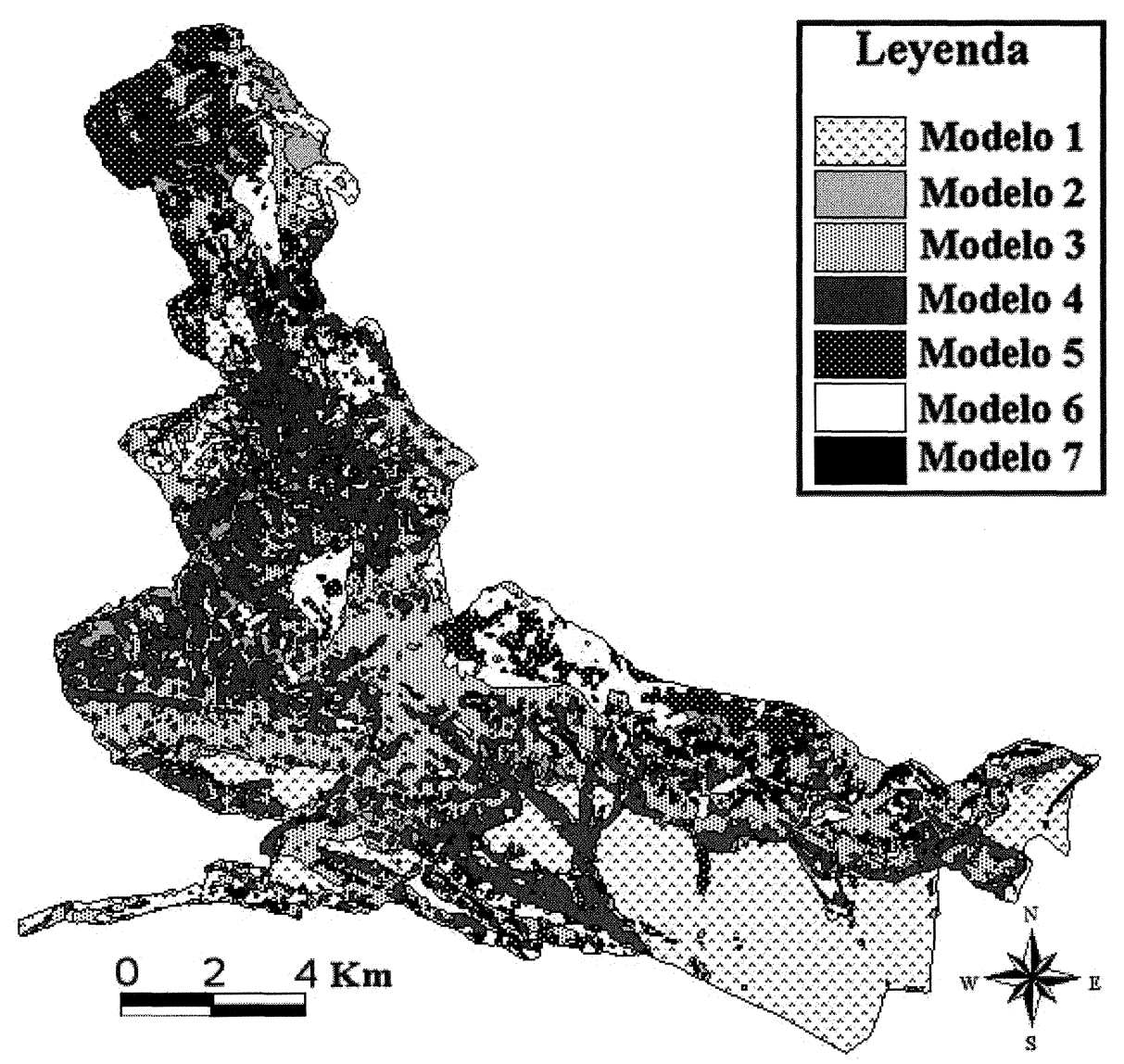

FIG. 4.-Mapa de modelos de combustible.

Cabañeros y donde hay más disponibilidad de nutrientes y agua aparece el modelo 4. El modelo 2 se encuentra en zonas donde el suelo no está muy desarrollado, llegando a no haber combustible en las zonas de alta pendiente donde aparecen manchas formadas por pedrizas. Finalmente, el modelo 1 ocupa la mayor parte de la Raña.

La matriz de confusión de la clasificación final (cuadro 1), con el 83\% de fiabilidad global, muestra cómo las mayores dificultades se presentaron en la discriminación entre las diferentes alturas del matorral (modelos 2, 3 y 4) y para identificar la presencia del matorral bajo el arbolado (modelos 5 y 6 ).

Estudios Geográficos, LXII, 243, 2001

$$
-325-
$$




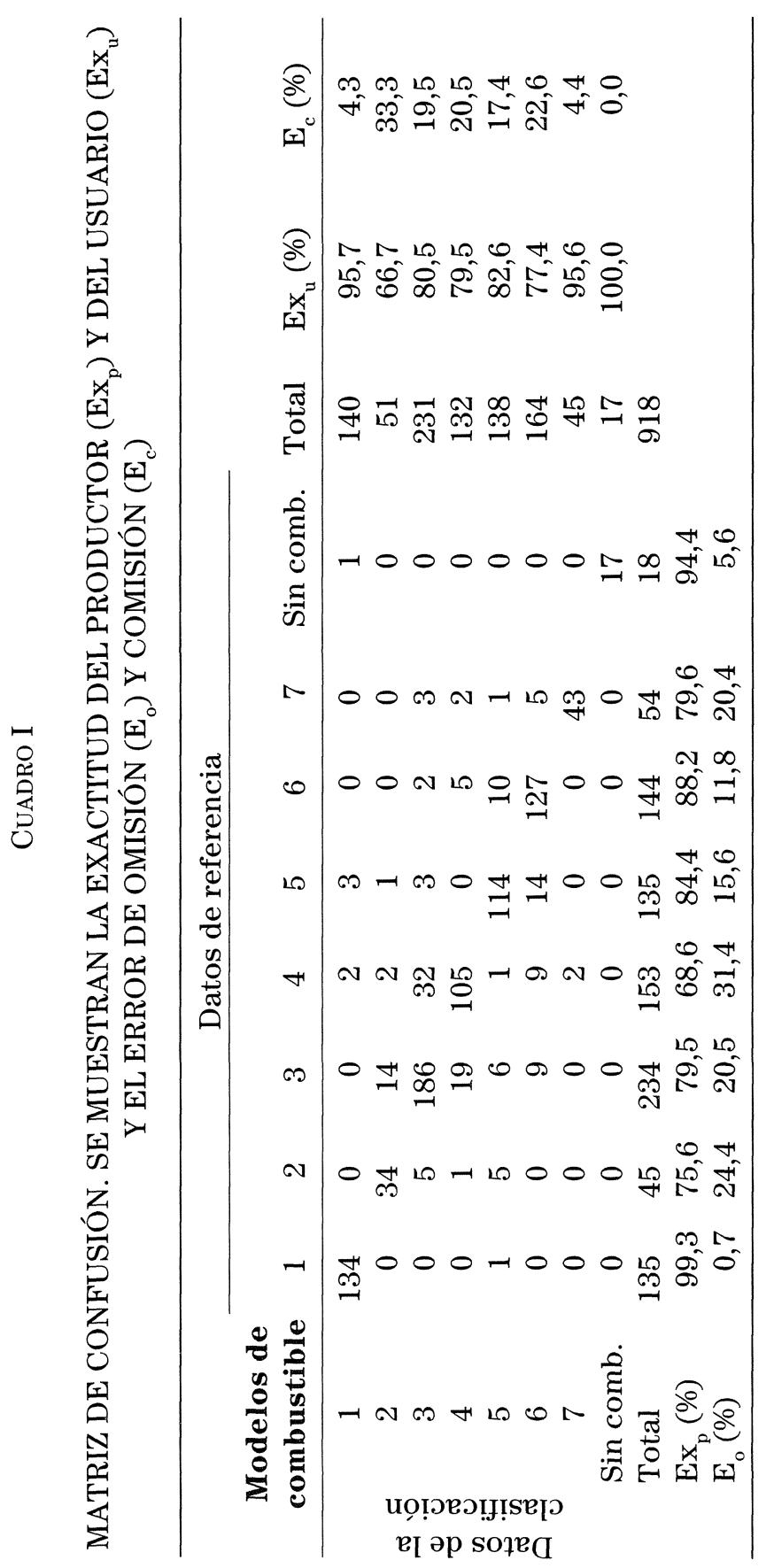

Estudios Geográficos, LXII, 243, 2001 
Los modelos de matorral se distinguen fundamentalmente por la altura y densidad, estando compuestos por especies similares; de ahí que la mayores dificultades de distinción aparecieran entre el modelo 3 y los modelos 2 y 4 . La confusión se redujo mucho entre el modelo 2 y 4 , ya que la diferencia de alturas y densidades entre ambos modelos es mucho mayor.

Los modelos de arbolado presentaron su mayor problema a la hora de diferenciar entre el modelo 5 y 6 . Estos dos modelos se distinguen por la presencia o no de sotobosque, lo que es difícil de identificar desde el espacio, debido a la presencia de los propios árboles. En las zonas de modelo 7 el espacio entre los árboles es mayor, con una mayor disponibilidad de luz para el crecimiento de matorral. Dentro de este modelo no aparecen pinares de repoblación. Ambas circunstancias, el mayor espaciado entre los árboles y la exclusión de los pinares, conllevaron su mejor distinción frente a los modelos 5 y 6 .

En lo que respecta a los errores de omisión, complementario de la exactitud del productor, la principal equivocación surgió con el modelo 4 , lo que implica que este modelo fue definido de una manera imperfecta, es decir que su delimitación resultó excesivamente reducida (Chuvieco, 1996). Por otra parte, el mayor error de comisión, complementario de la exactitud del usuario, aparece en el modelo 2, por lo que se evidenció una definición de este modelo demasiado amplia.

\section{Conclusiones}

La cartografía de modelos de combustible es una una de las variables más críticas en los sistemas de prevención y modelado de incendios forestales. Sin embargo, resulta de difícil acceso, ya que requiere intensivo trabajo de campo. La teledetección puede facilitar la disponibilidad de este tipo de cartografía, al permitir una visión exhaustiva y repetitiva del territorio. El ensayo que hemos realizado en el Parque Nacional de Cabañeros muestra, no obstante, algunas dificultades en el empleo de esta técnica, sobre todo las relacionadas con la distinción entre distintas alturas del matorral y la discriminación de éste cuando se encuentra bajo arbolado.

El ensayo realizado muestra el interés de utilizar información multitemporal, información auxiliar y correcciones radiométricas y topográficas que faciliten la discriminación de los modelos de combustible.

Estudios Geográficos, LXII, 243, 2001 
Las imágenes Landsat-TM resultaron apropiadas para la discriminación de los modelos de combustible. La textura y, sobre todo, las variables topográficas y la información multitemporal permiten incrementar la precisión de la clasificación.

El mapa de modelos de combustible generado mediante teledetección espacial puede ser integrado en los sistemas de prevención, predicción y modelado de incendios. Esta metodología podría ser útil para ser extrapolada a otras áreas de estudio. Queda todavía por resolver el problema de poder identificar las diferentes alturas de la vegetación y la presencia de sotobosque.

Como mejoras futuras en el empleo de esta técnica, cabe citar las siguientes:

- El principal reto está en solucionar el problema de la observación del sotobosque, que no puede observarse directamente salvo que la densidad del arbolado sea baja o se trate de especies caducifolias (en invierno). Se están ensayando algunas técnicas más novedosas como la interferometría radar y el láser (Blair et al., 1999), para intentar determinar la altura de la vegetación situada bajo los árboles.

- Determinación de la altura de la vegetación, que según Roberts $e t$ $a l$. (1997) puede llegar a estimarse a partir del componente puro que tiene en cuenta el porcentaje de sombra.

- Empleo de sensores de alta resolución espacial, como IKONOS (1 m), y de alta resolución espectral, como ASTER (14 bandas y $15 \mathrm{~m}$ ).

- Desarrollo de nuevos clasificadores (Wilson et al., 1994).

- Datos auxiliares de mayor calidad (Wilson et al., 1994).

- Localización más exacta, mediante GPS, de parcelas de entrenamiento y de verificación.

- Mayor inversión económica en trabajo de campo.

\section{AGRADECIMIENTOS}

Esta investigación ha sido financiada por los proyectos ControlFire-Sat e Inflame del Programa de la Comisión Europea Medio Ambiente y Clima (DG-XII). Deseamos expresar nuestro agradecimiento a las autoridades y guardería del Parque Nacional de Cabañeros por la ayuda prestada en la realización del trabajo de campo.

Estudios Geográficos, LXII, 243, 2001

$$
-328-
$$




\section{BIBLIOGRAFÍA}

AgEe, J. K. y PickFord, S. G. (1985). «Vegetation and fuel mapping of North Cascades National Park. Final Report». College of Forest Resources. Seattle.

Alonso, L. et al. (1997). «Parque Nacional de Cabañeros». Ecohábitat.

ANDERSON, H. E. (1982). «Aids to determining fuel models for estimating fire behavior»: General Technical Report INT-122. USDA, Forest Service. Ogden, UT.

Aranda, J. M., DE CASTro, A. J., Briz, S., Meléndez, J. y LÓPEZ, F. (1999). «Teledetección precoz de incendios forestales mediante sensores IR: Discriminación espectral de falsas alarmas». En: Teledetección. Avances y Aplicaciones (Castaño, S. y Quintanilla, A., (Eds)). Asociación Española de Teledetección. Albacete, España. pp. 271-274.

Benson, A., Greenlee, J. y Langenheim, J. (1982). «Assessment of Wildland Fuel Hazards in Big Basin Redwoods State Park, California». 5th International Symposium on Computer-Assisted Cartography. Crystal City, Virginia. pp. 55-63.

BLAIR, J. B., RABINE, D. L. y HoFTON, M. A. (1999). «The Laser Vegetation Imaging Sensor: a medium-altitude, digitisation-only, airborne laser altimeter for mapping vegetation anfd topography». ISPRS Journal of Photogrammetry \& Remote Sensing 54: 115-122.

Burgan, R. E., Klaver, R. W. y KlaVer, J. M. (1998). «Fuel models and fire potential from satellite and surface observations». International Journal of Wildland Fire 8 (3): 159-170.

Burgan, R. E. y Rothermel, R. C. (1984). «BEHAVE: Fire Behavior Prediction and Fuel Modeling System. Fuel Subsystem»: GTR INT-167. USDA Forest Service. Ogden, Utah.

BURGAN, R. E. y SHASBY, M. B. (1984). «Mapping broad-area fire potential from digital fuel, terrain, and weather data». Journal of Forestry 82: 228-231.

Cosentino, M. J. y Estes, J. E. (1981). «Use of Landsat data to develop a fuels database for a wildland fire simulation model». Pecora VII Symposium. Sioux Falls. pp. 590-599.

Cosentino, M. J., Woodcock, C., y Franklin, J. (1981). «Scene Analysis for Wildland FireFuel Characteristics in a Mediterranean Climate». 15th International Symposium on Remote Sensing of Environment. Ann Arbor, MI. pp. 635-646.

Chavez, P. S. (1996). «Image-based atmospheric corrections. Revisited and improved». Photogrammetric Engineering and Remote Sensing 62 (9): 1025-1036.

CHUvieco, E. (1996). «Fundamentos de Teledetección Espacial». Editorial Rialp. Madrid.

DEEMING, J. E. (1975). «Fuel models in the national fire danger rating system». Journal of Forestry 73 (6): 347-350.

DiXoN, R., SHIPLEY, R. y BRIGGS, A. (1984). «Landsat - a tool for mapping fuel types in the Boreal Forest of Manitoba. A pilot study». Manitoba Remote Sensing Center.

Fire Management and Comunications Section. Canada Centre for Remote Sensing. Winipeg, Manitoba.

ICONA (1990). «V Curso Superior sobre defensa contra incendios forestales». Madrid.

KouRTZ, P. H. (1977). «An application of Landsat digital technology to forest fire fuel type mapping». 11th International Symposium on Remote Sensing of Environment. Ann Arbor. pp. 1111-1115.

KYUN, I. A. (1997). «Tratamiento digital de imágenes Landsat5-TM y ERS-1 SAR para la clasificación de modelos de combustible». Trabajo de investigación. Universidad de Alcalá de Henares. Alcalá de Henares. 141 pp.

Estudios Geográficos, LXII, 243, 2001 
Martín, M. P., Chuvieco, E. y AGuado, I. (1998). «La incidencia del fuego en España». Serie Geográfica 7: 23-36.

MCKinley, R. A., Chine, E. P. y Werth, L. F. (1985). «Operational fire fuels mapping with NOAA-AVHRR data». En: Pecora 10. Remote sensing in forest and range resource management. Fort Collins, Colorado. U.S. Geological survey. pp. 295-304.

Miller, W. A., Howard, S. M. y Moore, D. G. (1986). «Use of AVHRR data in an information system for fire management in the Western United States». 20th International Symposium on Remote Sensing of Environment. Nairobi, Kenya. pp. 67-79.

Miller, W. A. y Johnston, D. C. (1985). «Comparison of Fire Fuel Maps Produced Using MSS and AVHRR Data». Pecora 10. Remote sensing in forest and range resource management. Fort Collins, Colorado. U.S. Geological survey. pp. 305-314.

PARdos CARRIón, J. A. y GonZÁlez Alonso, S. (1991). «Sequía, vegetación y fauna silvestre». Revista de la Real Academia de Ciencias Exactas, Físicas y Naturales 85 (2-3): 367385.

Pausas, J. G. y VAllejo, V. R. (1999). «The role of fire in European Mediterranean ecosystem». En: Remote Sensing of Large Wildfires in the European Mediterranean Basin (Chuvieco, E., (Ed.)) Springer-Verlag. Berlin. pp. 3-16.

PCI (1997). «Using PCI software». Editorial/Richmond Hill. Ontario (Canadá).

Peinado, M. y Martínez, J. M. (1985). «El Paisaje Vegetal de Castilla-La Mancha». Servicio de Publicaciones de la Junta de Comunidades de Castilla-La Mancha. Toledo.

PRIETO, F. (1995). «Incendios forestales. Aproximación a una propuesta preventiva, generadora de empleo, que actúe sobre sus causas y tendencias». Departamento de Ecología y Medio Ambiente de la Confederación Sindical de Comisiones Obreras.

Prometheus (1999). (http://kentauros.rtd.algo.com.gr/promet/schedule.htm).

RABII, H. A. (1979). «An investigation of the utility of Landsat-2 MSS data to the fire-danger rating area, and forest fuel analysis within Crater Lake National Park». Oregon State University.

RIAÑo, D. (2000). «Cartografía de modelos de combustible con teledetección». Trabajo de investigación. Universidad de Alcalá de Henares. Alcalá de Henares. 187 pp.

Roberts, D., Gardner, M., Regelbrugge, J., Pedreros, D. y Ustin, S. (1997). «Mapping the distribution of Wildfire fuels using AVIRIS in the Santa Monica Mountains». Summaries of the Seventh JPL Airbone Earth Science Workshop. Pasadena, California. Jet Propulsion Laboratory. pp. 345-352.

Root, R. R., Stitt, S. C. F., Nyquist, M. O., G. S., W. y Agee, J. K. (1986). «Vegetation and fire fuel models mapping of North Cascades National Park». ACSM-ASPRS Annual Convention. Washington, D.C. pp. 75-85.

Rosenfeld, A. y PFALTZ, J. L. (1966). «Sequential operations in digital picture processing». Journal of the association for computing machinery 13 (4): 471-494.

Rothermel, R. C. (1972). «A Mathematical Model for Predicting Fire Spread in Wildland Fuels»: Research Paper INT-115. USDA, Forest Service. Ogden, Utah.

SALAS, F. J. (1994). «Detección de áreas de riesgo de incendio forestal a partir de los Sistemas de Información Geográfica y la Teledetección.». Tesis doctoral. Universidad de Alcalá de Henares. Alcalá de Henares. 471 pp.

SALAS, F. J. y Chuvieco, E. (1995). «Aplicación de imágenes Landsat-TM a la cartografía de modelos combustibles». Revista de Teledetección 5: 18-28.

Estudios Geográficos, LXII, 243, 2001

$$
-330-
$$


SAlAZAR, L. A. (1982). «Remote Sensing Techniques Aid in Preattack Planning for Fire Management»: Res. Paper PSW-162. USDA, Forest Service. Berkeley, CA.

Shasby, M. B., Burgan, R. R. y Johnson, G. R. (1981). «Broad area forest fuels and topography mapping using digital Landsat and terrain data». Machine Processing of Remotely Sensed Data Symposium. West Lafayette. Purdue University. pp. 529-538.

STоw, D. A. (1993). «The role of Geographic Information Systems for landscape ecological studies». En: Landscape Ecology and Geographic Information Systems (HainesYoung, R., Green, D.R. y Cousins, S.H., (Eds)). Taylor and Francis. London. pp. 11-21.

Teillet, P. M., Guindon, B. y Goodeonugh, D. G. (1982). «On the slope-aspect correction of multispectral scanner data». Canadian Journal of Remote Sensing 8 (2): 84-106.

Tymstra, C. y EllehoJ, E. A. (1994). «Fire behavior prediction fuel type mapping using the Alberta Vegetation Inventory». 8th Annual symposium on geographic information systems in forestry, environmental and natural resources management. Vancouver, British Columbia. Polaris Conferences.

VAN WANGTENDONK, J. W. (1997). «Use of thematic mapper imagery to map fuel models». 13th Conference on Fire and Forest Meteorology. Lorne, Australia. International Association of Wildland Fire.

VAN WANGTENDONK, J. W. y RoOT, R. R. (1999). «Hyperspectral analysis of multitemporal Landsat-TM data for mapping fuels in Yosemite National Park». The Joint Fire Science conference and Workshop - Crossing the Millenium: Integrating Spatial Technologies and Ecological Principles for a New Age in Fire Management. Boise, Idaho.

Vasconcelos, M. J. P., Paúl, J. C. U., Silva, S., Pereira, J. M. C., Caetano, M. S., Catry, F. X. y OliveiRA, T. M. (1998). «Regional fuel mapping using a knowledge based system approach». III International Conference on Forest Fire Research-14th Conference on Fire and Forest Meteorology. Coimbra. ADAI. pp. 2111-2123.

WeRTH, L. F., MCKinley, R. A. y ChINE, E. P. (1985). «The use of wildland fire fuel maps produced with NOAA-AVHRR scanner data». En: Pecora X Symposium Fort Collins. pp. 326-331.

Wilson, B. A., Ow, C. F. Y., Heathcott, M., Milne, D., McCaffrey, T. M., Ghitter, G. y FranKLIN, S. E. (1994). «Landsat MSS classification of fire fuel types in Wood Buffalo National Park, Northern Canada». Global Ecology and Biogeography Letters 4: 33-39.

Wukelic, G. Z., Gibbons, D. E., Martucci, L. M. y Foote, H. P. (1989). «Radiometric calibration of Landsat Thematic Mapper thermal band». Remote Sensing of Environment 28: 339-347.

Yool, S. R., Eckhardt, D. W., Cosentino, M. J. y Estes, J. E. (1984). «A Geographic Information System approach to quantitative assessment of wildlife fuels». ACSM-ASPRS Annual Convention. Washington DC. pp. 792-801.

RESUMEN: La conjunción entre desarrollo y sostenibilidad del medio ambiente se torna un aspecto de singular importancia en nuestra sociedad. La incidencia de la actividad humana en la aparición de incendios forestales supone un ejemplo nítido de esa afirmación, ya que los cambios recientes en los patrones de utilización del territorio van acompañados de un notable aumento de los incendios forestales, con la consiguiente degradación del suelo y la cubierta vegetal. En este contexto, resulta de suma importancia 
mejorar las herramientas de prevención de incendios y evaluación de sus efectos, de cara a reducir la ocurrencia o paliar los efectos de este fenómeno.

En este artículo analizamos el papel de la teledetección espacial para obtener cartografía de modelos de combustible. Estos mapas aportan una información básica en los sistemas de prevención, predicción y modelado de incendios forestales, ya que permiten analizar las características y estructura de la vegetación de cara a la propagación del fuego. Hemos utilizado como área de estudio el Parque Nacional de Cabañeros (Ciudad Real). La cartografía de combustible se ha basado en el análisis de dos imágenes de satélite Landsat-TM, junto con información auxiliar (modelos digitales de elevación y texturas). Los resultados de la clasificación ofrecieron una precisión promedio del $83 \%$. Las mayores dificultades que encontramos hacen referencia a la discriminación entre las diferentes alturas del matorral y la identificación del matorral bajo el arbolado.

PAlABRAS Clave: Cartografía de modelos de combustible, teledetección espacial, incendios forestales, desarrollo sostenible.

ABSTRACT: Fuel type mapping from remote sensing data: Contributions to a sustainable environmental development. The conjugation of human development and sustainability of natural resources is a critical issue to our present society. The role of human activity in fire ocurrence is a good example of the difficult dialogue between development and conservation. Recent changes in land use patterns have carried along an increasing rate of fire occurrence, which implies in some cases severe effects on soil erosion and vegetation degradation. For this reason, it is critical to improve current tools to prevent and assess forest fires, so that fire occurrence may be reduced.

This paper explores the use of remote sensing methods to derive fuel type maps. These maps are basic sources of information for fire behaviour modelling, since they make it possible to take into account vegetation characteristics for fire propagation. The study area is located in Cabañeros National Park (Central Spain). The fuel type mapping was based in the analysis of two satellite Landsat-TM images and auxiliary information (digital elevation models and texture images). The digital classification worked out to have a global accuracy of $83 \%$. The larger problems found were to distinguish between the different heights of shrubs and identifying the existence of shrubs under the tress.

KEYwoRDS: Fuel type mapping, remote sensing, forest fires, sustainable development.

RÉSUME: La cartographie des modèles de combustible avec télédétection: apports pour développement environnemental durable. La conjonction entre développement et soutenance de l'environnement devient un aspect de singulière importance dans notre société. L'incidence de l'activité humaine dans l'apparition des incendies forestiers suppose un exemple net de cette affirmation, puis que les récents changes des modèles d'utilisation du territoire vont accompagnés d'une notable augmentation des incendies forestiers, avec la conséquente dégradation du sol et la couverture végétale. Dans ce contexte suite de

Estudios Geográficos, LXII, 243, 2001

$$
-332-
$$


somme importance améliorer les outils de prévention des incendies et l'évaluation de ses effets, pour réduire l'incidence ou pallier les effets de ce phénomène.

Dans cet article, on présente un exemple de ce tipe de vises. En concret, nous analysons le papier de la télédétection spatiale pour obtenir cartographie des modèles de combustible. Ces cartes apportent une information basique dans les systèmes de prévention, prédiction et modelage des incendies forestiers, puis que permettent analyser les caractéristiques et les structures de la végétation vers la propagation du feu. Nous avons utilisé comme surface d'étude le Parc National de Cabañeros (Ciudad Real). La cartographie de combustible s'est appuyée sur l'analyse de deux images de satellite Landsat-TM et renseignement auxiliaire (modèles digitales d'élévation et textures). Les résultats de la classification offrent une précision d'un $83 \%$. Les difficultés les plus grandes que présente cette technologie sont la discrimination entre les hauteurs différentes de l'arbuste et l'identification de ceux-ci sous des arbres.

Des Mots Clefs: Cartographie de modèles de combustible, télédétection spatiale, incendies forestiers, développement durable. 